\title{
CLINICAL PROFILE OF HAEMOPHILIA PATIENTS OF UPPER ASSAM- A HOSPITAL-BASED STUDY
}

\author{
Anupam Dutta1, Taniya Sarkar Dutta ${ }^{2}$, Pranoy Dey ${ }^{3}$ \\ ${ }^{1}$ Assistant Professor, Department of Medicine, Assam Medical College and Hospital. \\ ${ }^{2}$ Registrar, Department of Paediatrics, Assam Medical College and Hospital. \\ ${ }^{3}$ Associate Professor, Department of Paediatrics, Assam Medical College and Hospital.
}

ABSTRACT

\section{BACKGROUND}

Haemophilia is a rare condition where patients present with abnormal bleeding and other complications of bleeding due to genetic or acquired deficiency of coagulation factor VIII (Haemophilia A) or Factor IX (Haemophilia B).

Aims and Objectives- We have studied the clinical profile of haemophilia patients who have come to our tertiary medical centre for hospitalisation with some complication.

\section{MATERIALS AND METHODS}

All patients who were admitted in the Department of Medicine and Paediatrics with Haemophilia were included in the study. Detail clinical and family history was taken followed by physical examination and necessary investigations. Factor replacement was done using our institutional protocol, which is based on the Significant Resource Constraint Guidelines of Haemophilia Federation India.

\section{RESULTS}

A total of 79 haemophilia patients were studied with $94.9 \%$ males and 4 females; $74.7 \%$ were below the age of 20 years and $78.5 \%$ were known haemophilia patients, whereas $21.5 \%$ were newly diagnosed; $64.6 \%$ patients had a definite family history; $79.7 \%$ were factor VIII deficient and 20.3\% were factor IX deficient; 47 (74.6\%) patients had severe factor VIII deficiency, 12 (19\%) patients had moderate factor VIII deficiency and 4 (6.4\%) patients had mild factor VIII deficiency; 11 (68.7\%) patients had severe factor IX deficiency. Out of the 57 patients who presented with joint bleed $38(67 \%)$ had knee joint involvement, 16 (28\%) had ankle joint involvement, 13 (22.8\%) had wrist involvement, 8 (14\%) had elbow involvement and 3 (5.3\%) had shoulder joint bleed. Out of the 19 cases of intramuscular bleed, 13 (68.4\%) patients had psoas bleed; 43 (75.4\%) patients had multiple joint involvement; 7 (12.3\%) patients both joint bleed and intramuscular bleed; 68.4\% patients had joint deformity. Out of the four females with mild-to-moderate factor VIII deficiency, two had joint bleed and two had uncontrolled menstrual bleed.

\section{CONCLUSION}

Most of our patients were below 20 years (74.7\%) and haemophilia A (79.7\%). One-third of them did not have a family history. Most of them had a severe factor deficiency and knee joint was the most common joint to bleed. Two-third of the patients had joint deformity.

\section{KEYWORDS}

Haemophilia A, Haemophilia B, Haemarthrosis, Factor Deficiency VIII and IX.

HOW TO CITE THIS ARTICLE: Dutta A, Dutta TS, Dey P. Clinical profile of haemophilia patients of upper Assam- a hospital-based study. J. Evolution Med. Dent. Sci. 2017;6(37):2990-2993, DOI: 10.14260/Jemds/2017/645

\section{BACKGROUND}

Haemophilia is a rare condition where patients present with abnormal bleeding and other complications of bleeding due to genetic or acquired deficiency of coagulation factor VIII (Haemophilia A) or factor IX (Haemophilia B). It is frequently encountered in families, where it passes from one generation to another as an X-linked recessive disease, mostly involving boys whereas girls are mostly carriers. In up to $30 \%$ cases no family history is present, hence representing spontaneous mutations. The life expectancy is grossly reduced and the suffering along with risk of complication is tremendously increased in those patients who cannot afford prophylactic factor replacement therapies.

Financial or Other, Competing Interest: None. Submission 01-04-2017, Peer Review 24-04-2017,

Acceptance 01-05-2017, Published 08-05-2017.

Corresponding Author:

Dr. Anupam Dutta,

Revti House, Purnananda Road,

Shantipara, Dibrugarh-786001,

Assam.

E-mail: dranupamdutta80@gmail.com

DOI: $10.14260 /$ jemds $/ 2017 / 645$

\section{(c) $(1)$}

\begin{abstract}
Aims and Objectives
We have studied the clinical profile of haemophilia patients who have come to our tertiary medical centre for hospitalisation for some complication over a period of 18 months (from $1^{\text {st }}$ October 2015 till 31 $1^{\text {st }}$ March 2017).

\section{MATERIALS AND METHODS}

All patients who were admitted in the Department of Medicine and Paediatrics with Haemophilia were included in the study. Detail clinical and family history was taken followed by physical examination and necessary investigations. Factor replacement was done using our institutional protocol, which is based on the significant resource constraint guidelines of World Federation of Haemophilia for the Management of Haemophilia. ${ }^{1}$
\end{abstract}

\section{RESULTS}

A total of 79 haemophilia patients were included in our study, which included 75 (94.9\%) males and 4 (5.1\%) females; 59 $(74.7 \%)$ haemophilia patients were below the age of 20 years (Figure 1); $16(20.2 \%)$ patients were below the age of 5 years, $13(16.5 \%)$ patients were from 6 to 10 years, 18 $(22.8 \%)$ patients were from 11 to 15 years, $16(20.2 \%)$ 
patients were from 16 to 20 years, 7 (8.9\%) patients were from 21 to 25 years and 9 (11.4\%) patients were above 25 years in age; 62 (78.5\%) patients were known haemophilia patients and were on frequent followup, whereas 17 (21.5\%) patients were newly diagnosed with haemophilia (Figure 2). Four (4) cases were excluded from the study, which included two (2) cases that died with suspected haemophilia and two (2) cases that could not afford any investigations and took discharge against medical advice; $51(64.6 \%)$ patients had a definite family history of haemophilia, whereas 28 (35.4\%) patients had no family history; $4(5 \%)$ patients have a history of consanguineous marriage in their family.

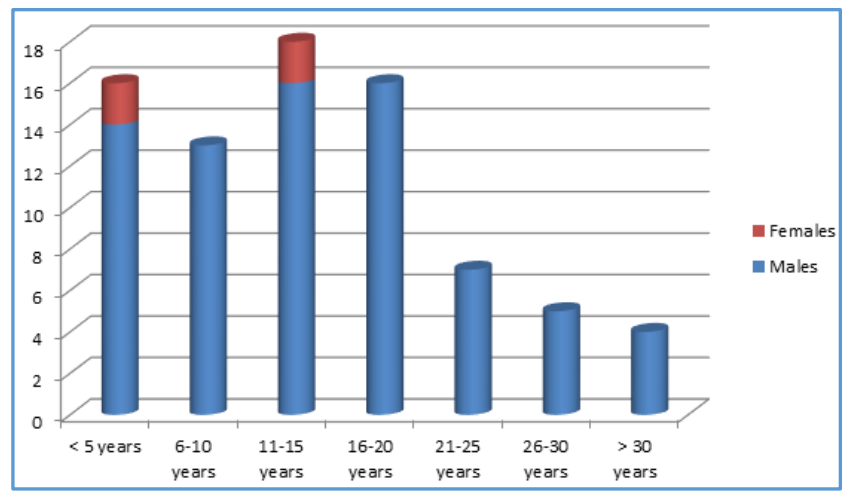

Figure 1. Demographic Profile of Haemophilia Patients Hospitalised in our Institute

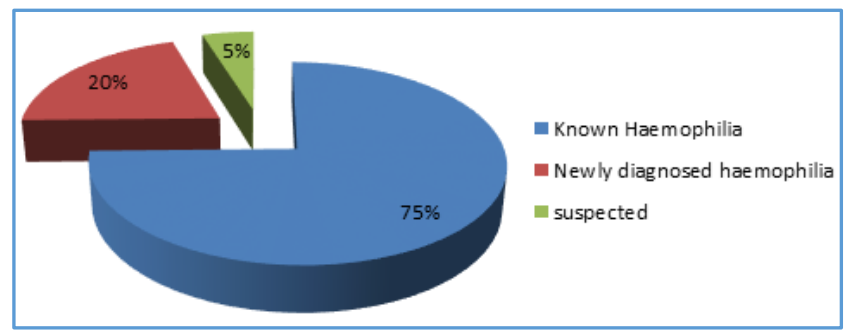

Figure 2. Known and Newly Diagnosed cases of Haemophilia who were Hospitalised

$63(79.7 \%)$ cases were factor VIII deficient i.e. Haemophilia A and 16 (20.3\%) cases were factor IX deficient i.e. Haemophilia B (Figure 3); 47 patients had severe factor VIII deficiency, 12 patients had moderate factor VIII deficiency and 4 patients had mild factor VIII deficiency; 11 patients had severe factor IX deficiency, 4 patients had moderate factor IX deficiency and 1 patient had mild factor IX deficiency (Figure 4).

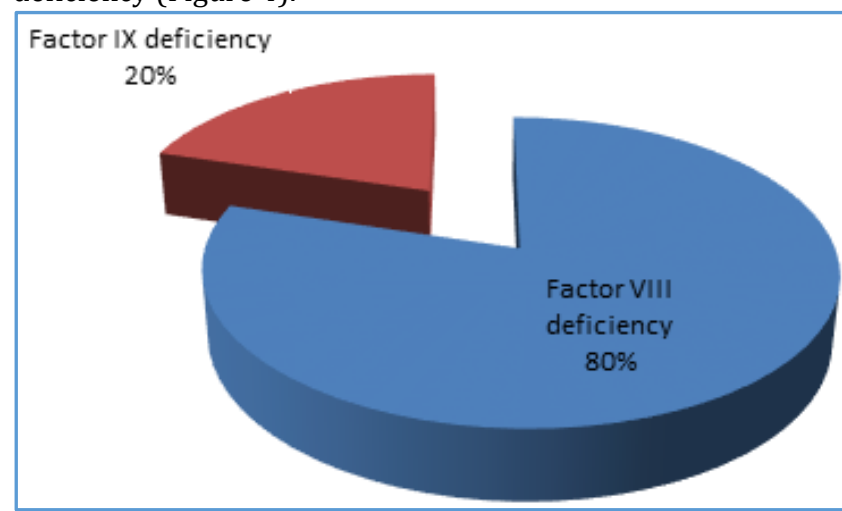

Figure 3. Percentage of Patients with Haemophilia A and Haemophilia B

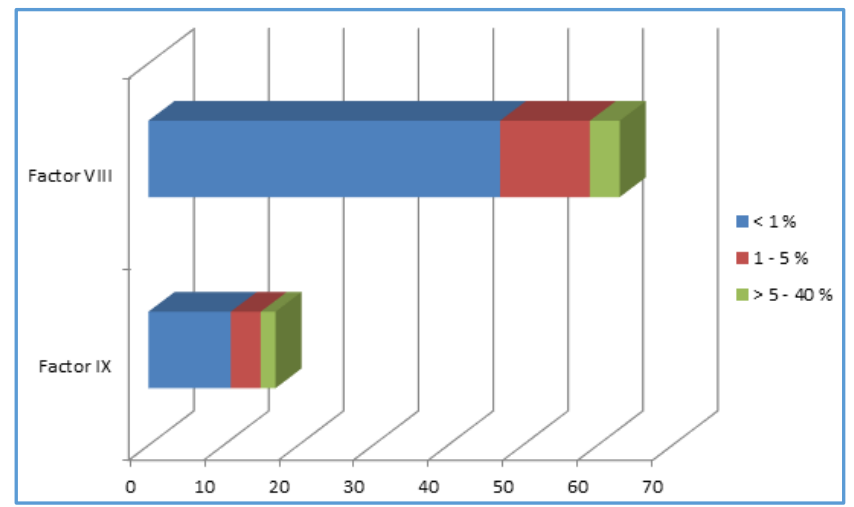

Figure 4. Profile of Factor Deficiencies with Severity (\% of Factor)

Most common chief complaint (43\%) and clinical feature $(72 \%)$ was joint bleed followed by intramuscular bleed (24\%) (Table 1). Other clinical presentations included teeth and gum bleed, haematuria, epistaxis, trauma, burn and superficial cuts and bruises. Four patients came with lifethreatening emergencies, which included two cases of intracranial bleed and two cases of intra-abdominal bleed. All these four cases were treated successfully by adequate factor replacement and necessary intensive care management. Two known haemophilic females attained menarche and came with uncontrolled menstrual bleeding, which got corrected with calculated factor replacement. They were counselled and are still on regular followup.

\begin{tabular}{|c|c|c|c|c|c|c|c|}
\hline Sl. No. & Characteristics & \multicolumn{4}{|c|}{ Number } & \multicolumn{2}{|c|}{ Percentage (\%) } \\
\hline \multicolumn{8}{|c|}{ Factor Deficiency } \\
\hline 1 & Factor VIII (Haemophilia A) & \multicolumn{4}{|c|}{63} & \multicolumn{2}{|c|}{$79.7 \%$} \\
\hline 2 & Factor IX (Haemophilia B) & \multicolumn{4}{|c|}{16} & \multicolumn{2}{|c|}{$20.3 \%$} \\
\hline \multicolumn{2}{|r|}{ Clinical Presentation } & Chief Complaint & $\%$ & Associated Manifestations & $\%$ & Total & $\%$ \\
\hline 1 & Joint Bleed (Haemarthrosis) & 34 & $43 \%$ & 23 & $29 \%$ & 57 & $72 \%$ \\
\hline 2 & Intramuscular bleed & 17 & $21.5 \%$ & 2 & $2.5 \%$ & 19 & $24 \%$ \\
\hline 3 & Intra-abdominal bleed & 2 & $2.5 \%$ & 0 & 0 & 2 & $2.5 \%$ \\
\hline 4 & Teeth and Gum bleed & 12 & $15.2 \%$ & 1 & $1.3 \%$ & 13 & $16.5 \%$ \\
\hline 5 & Haematuria & 1 & $1.3 \%$ & 0 & 0 & 1 & $1.3 \%$ \\
\hline 6 & Epistaxis & 6 & $7.6 \%$ & 1 & $1.3 \%$ & 7 & $8.9 \%$ \\
\hline 7 & Burn/Superficial bleed & 2 & $2.5 \%$ & 5 & $6.3 \%$ & 7 & $8.9 \%$ \\
\hline 8 & Trauma & 1 & $1.3 \%$ & 0 & 0 & 1 & $1.3 \%$ \\
\hline 9 & Intracranial bleed & 2 & $2.5 \%$ & 0 & 0 & 2 & $2.5 \%$ \\
\hline 10 & Uncontrolled menstrual bleed & 2 & $2.5 \%$ & 0 & 0 & 2 & $2.5 \%$ \\
\hline
\end{tabular}


Out of the 57 patients with joint bleed who presented with joint bleed, 38 (67\%) had knee joint involvement, 16 (28\%) had ankle joint involvement, 13 (22.8\%) had wrist involvement, 8 (14\%) had elbow involvement and $3(5.3 \%)$ had shoulder joint bleed (Figure 5). Out of the 19 cases of intramuscular bleed $13(68.4 \%)$ patients had psoas bleed, 3 (15.8\%) patients had bleed in biceps and $2(10.5 \%)$ had bleed in triceps and $2(10.5 \%)$ had bleed in forearm; 43 (75.4\%) patients had multiple joint involvement; 7 (12.3\%) patients both joint bleed and intramuscular bleed; 39 (68.4\%) patients had joint swelling and deformity.

After joint and intramuscular bleed, the most common presentation was uncontrolled bleeding from teeth and gums which was present in $13(16.5 \%)$ patients followed by epistaxis and superficial bleed which were both present in 7 $(8.9 \%)$ each. Teeth and gum bleed and epistaxis were mostly presented as chief complaint, whereas superficial bleed was mostly an associated complaint.

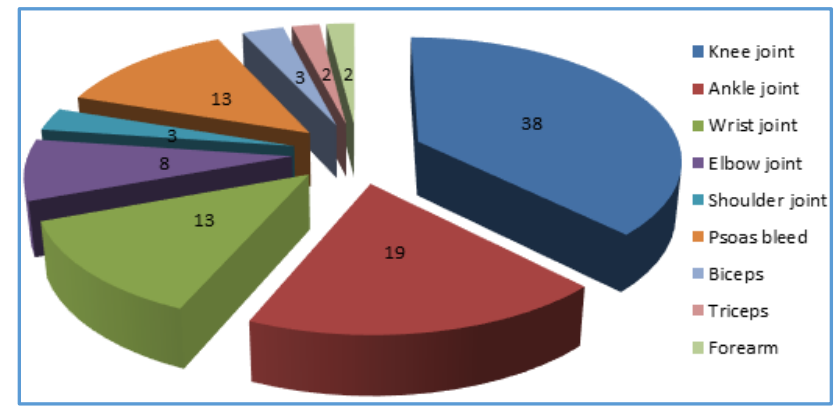

Figure 5. Profile of Joint Bleed and Intramuscular Bleed in Haemophilia Patient

We had four females in our study. One was three-year-old girl with right elbow bleed and another was six years with right knee bleed and left ankle bleed. Two 13-year-old girls who were our followup case for some time presented with uncontrolled bleeding during menarche. One of them also had uncontrolled bleeding from gums and teeth. One of them had a family history of haemophilia and consanguineous marriage in the family. Rest of the three cases were sporadic with no family history. All of them were mild-to-moderate factor VIII deficient patients.

\section{DISCUSSION}

Haemophilia has an average incidence of 1 per 10,000 births and the estimated number of patients with haemophilia in India is $77,000 .^{2}$ Indian data as reported by Haemophilia Federation of India showed 14,718 patients with bleeding disorders and 11,586 patients with Haemophilia A in the year $2011 .{ }^{3}$ Out of the top five countries with highest number of reported haemophilia (USA, India, Brazil, China and UK) patients, India has the second highest number of haemophilia patients.

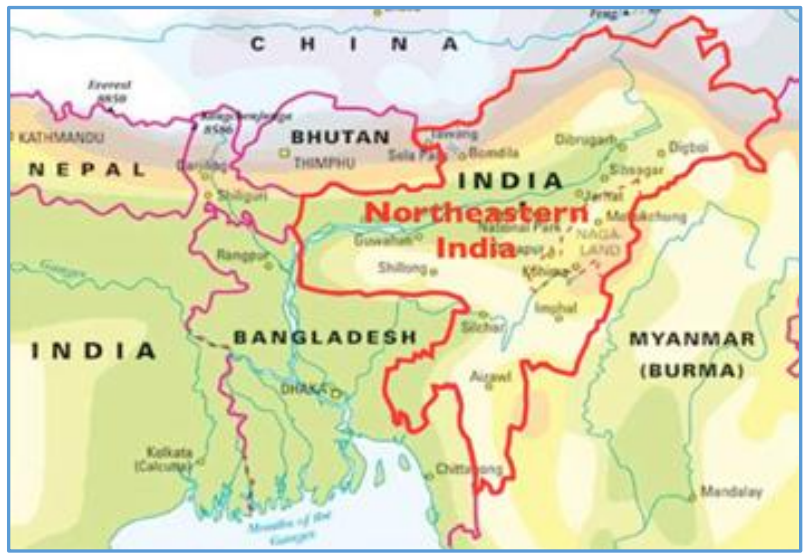

Image 1. Geographic Location of our Centre

North East India comprises of Arunachal Pradesh, Assam, Manipur, Meghalaya Mizoram, Nagaland, Sikkim and Tripura. Out of these, our tertiary centre caters to eastern Assam, eastern Arunachal Pradesh and some parts of Nagaland. Our centre is also supported by Haemophilia Federation India (HFI) with free factors (Image 2). So, we provide free factors to patient from Upper Assam and nearby states like Arunachal Pradesh and Nagaland who do not have any factor support or haemophilia care centre.

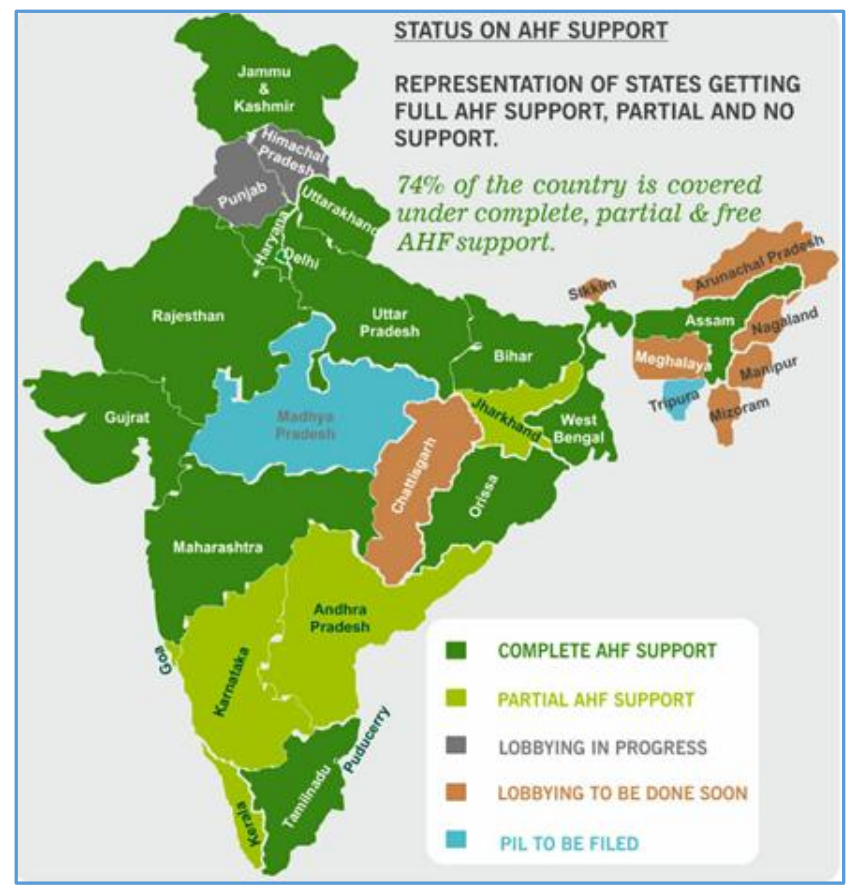

Image 2. Status of Anti-Haemophilia Factors support by Haemophilia Federation India (HFI) 2017

As haemophilia is a rare disease, demographic data and clinical profiling of these patients are very limited, especially in a developing country like India. In one of our earlier studies we had reported 45 documented cases with 36 (81\%) factor VIII deficiency, 8 (17\%) factor IX deficiency and one factor X deficiency case. ${ }^{4}$ Kar A et al studied 1467 patients from Maharashtra and found the ratio of haemophilia A to haemophilia B patients was 4.2:1.5 Mishra S et al in a study from western Uttar Pradesh reported that type A haemophilia was observed in $88.3 \%(68 / 71)$ patients. $^{6}$ We found 63 
(79.7\%) cases with factor VIII deficiency and 16 (20.3\%) cases with factor IX deficiency.

Jayandharan G et al analysed DNA from 109 unrelated Indian patients with Haemophilia A (HA) for their FVIII gene defects. Among these patients 89 (82\%) had severe (FVIII: C $<1 \%)$ HA, $11(10 \%)$ had moderate (FVIII: C 1-5\%) HA and 9 (8\%) had mild (FVIII: C 5 - 30\%) HA.7 In our early study we found that out of 36 factor VIII deficient patients, 17 (47.2\%) had severe, $8(22.2 \%)$ had moderate and $11(30.6 \%)$ had mild factor VIII deficiency. ${ }^{4}$ Kar $\mathrm{A}$ et al reported that majority (85\%) of the haemophilia patients studied had severe FVIII deficiency. ${ }^{5}$ Mishra S et al also noted that majority of their patients had severe haemophilia $(80.5 \%){ }^{6}$

In our present study also, 47 (74.6\%) patients had severe factor VIII deficiency, 12 (19\%) patients had moderate factor VIII deficiency and $4(6.4 \%)$ patients had mild factor VIII deficiency; $11(68.7 \%)$ patients had severe factor IX deficiency, 4 (25\%) patients had moderate factor IX deficiency and one patient had mild factor IX deficiency.

Bhattacharya DK et al also noted that nearly one-third of cases of haemophilia occur with no preceding family history, possibly from new genetic mutation. ${ }^{8}$ Mishra $S$ et al had noted that the family history was present in $58.4 \%$ of the patients. ${ }^{6}$ We found that $51(64.6 \%)$ patients had a definite family history of haemophilia, whereas 28 (35.4\%) patients had no family history.

Mishra $\mathrm{S}$ et al also reported that joint involvement was present in $77.9 \%$ and knee joint was observed to be as the target joint among $57.1 \%$ of the patients; $76.6 \%$ of the patients had joint swelling resulting in compromised joint movement. Bleeding occurred in soft tissues and joints in $62.3 \%$ and $15.6 \%$ of the patients, respectively. ${ }^{6}$ We found joint involvement in $72 \%$ and as a chief complaint in $43 \%$. Out of them $67 \%$ had knee joint involvement, $28 \%$ had ankle joint involvement, $22.8 \%$ had wrist involvement; $75.4 \%$ patients had multiple joint involvement; $12.3 \%$ patients both joint bleed and intramuscular bleed; $68.4 \%$ patients had joint swelling and deformity.

\section{CONCLUSION}

Most of our patients were below 20 years (74.7\%) and haemophilia A (79.7\%). One-third of them did not have a family history. Most of them had a severe factor deficiency and knee joint was the most common joint to bleed. Twothird of the patients had joint deformity.

\section{ACKNOWLEDGEMENTS}

We are thankful to Prof. Anup Kumar Das, Head of the Department of Medicine, AMCH and Prof. Helena Rahman, Head of the Department of Paediatrics, AMCH for allowing us to conduct this study. We are extremely thankful to Haemophilia Federation India for providing our patients with Factor VIII and IX free of cost.

\section{REFERENCES}

[1] Srivastava A, Brewer AK, Mauser-Bunschoten EP, et al. Guidelines for the management of Hemophilia. Hemophilia 2012:1-47.

[2] Kar A, Phadnis S, Dharmarajan S, et al. Epidemiology and social costs of hemophilia in India. Indian J Med Res 2014;140(1):19-31.

[3] World Federation of Hemophilia (WFH). Report on the Annual Global Survey 2011. Canada: WFH, 2013. http://www1.wfh.org/publications/files/pdf-488. pdf

[4] Dutta A, Kar S, Kakati S, et al. Study on clinical presentations of factor deficient patients presenting to a tertiary care centre of North East India. Journal of Medical Science and Clinical Research 2016;4(7). ISSN(e)-2347-176, ISSN (p)- 2455-0450.

[5] Kar A, Potnis-Lele M, Descriptive epidemiology of hemophilia in Maharashtra, India. Hemophilia 2001;7(6):561-7.

[6] Mishra S, Kumar S, Panwar A, et al. A clinical profile of hemophilia patients and assessment of their quality of life in Western Uttar Pradesh, India: an observational study. Med J DY Patil Univ 2016;9:320-4.

[7] Jayandharan G, Shaji RV, Baidya S, et al. Identification of factor VIII gene mutations in 101 patients with haemophilia A: mutation analysis by inversion screening and multiplex PCR and CSGE and molecular modelling of 10 novel missense substitutions. Hemophilia 2005;11(5):481-91.

[8] Bhattacharya DK. Hemophilia in the Indian Scenario. Int J Hum Genet 2006;6(1):33-9. 